\title{
Utilizar las matemáticas para resolver problemas reales
}

\author{
SANTIAGO VICENTE*, WIM VAN DOOREN** \\ Y LIEVEN VERSCHAFFEL ** \\ *Universidad de Salamanca; **Katholieke Universiteit Leuven
}

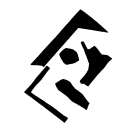

Resumen

En este trabajo de revisión se analizan las razones por las que los alumnos no son capaces de resolver problemas realistas utilizando conocimientos no matemáticos. Para ello se describen, en primer lugar, las investigaciones internacionales que han documentado estas dificultades en la resolución de problemas realistas. En segundo lugar, se describe cómo los libros de texto y la cultura del aula favorecen que los niños vayan aprendiendo de manera progresiva a resolver los problemas de matemáticas utilizando únicamente sus conocimientos matemáticos, y cómo este aprendizaje va abriendo una brecha entre las matemáticas escolares y el mundo real. En tercer lugar, se describe un estudio instruccional que ha demostrado que lograr que los alumnos resuelvan problemas realistas sin ceñirse exclusivamente a sus conocimientos matemáticos es un objetivo factible y deseable. Finalmente, se exponen una serie de consideraciones acerca del esfuerzo que supone este tipo de enseñanza de la matemática y la resolución de problemas y de los limites que marcan esta perspectiva del proceso de enseñanza y aprendizaje de las matemáticas.

Palabras clave: Resolución de problemas, problemas realistas, cultura de aula.

\section{Using mathematics for solving real problems}

\begin{abstract}
This paper analyses the reasons underlying children's difficulties when solving realistic wor(l)d problems in the mathematics class. In order to do so, first, international studies on these difficulties are reported. Second, we describe how characteristics of the current textbooks and classroom interaction led children to learn that mathematical problems must be solved by using only mathematical information and procedures, and not by attending to relevant real-world aspects of these problems, and how this learning process moves away the mathematics of the school from the real life. Third, an intervention study that showed bow the classroom practice and culture can be successfully modified is described. Finally, some comments about the limits of these ideas are made.
\end{abstract}

Keywords: Word problem solving, realistic word problems, classroom culture.

Agradecimientos: Este trabajo ha sido financiado por el proyecto SEJ2006/11249, del Ministerio de Educación y Ciencia y por el proyecto GOA 2006/01 "Developing adaptive expertise in mathematics education" de la Research Fund de la K. U. Leuven, Bélgica.

Correspondencia con los autores: Santiago Vicente. Universidad de Salamanca. Facultad de Educación. Pso. Canalejas 169.37008 Salamanca. E-mail: sanvicente@usal.es

Original recibido: Julio, 2008. Aceptado: Septiembre, 2008. 
Durante la escolaridad obligatoria una de las habilidades instrumentales, a cuya adquisición los maestros y los alumnos dedicarán una cantidad muy considerable de tiempo, son las matemáticas. De hecho, la capacidad de aprender nuevos contenidos durante la Educación Secundaria dependerá de la adquisición de esta habilidad instrumental en los primeros años de escolaridad. En el transcurso de este aprendizaje los alumnos deberán adquirir, por un lado, la capacidad de ejecutar rápida y eficazmente operaciones aritméticas, y por otro, la de resolver problemas. Estas dos habilidades son complementarias entre sí, ya que mientras que la ejecución de operaciones permitirá a los alumnos disponer de procedimientos matemáticos para resolver situaciones problemáticas, la resolución de problemas les permitirá encontrar contextos en los que aplicar esas operaciones, dotándolas así de utilidad y de sentido. Sin embargo, mientras que los alumnos suelen no presentar dificultades al ejecutar operaciones, a menudo sí las presentan cuando resuelven problemas, especialmente aquellos que no se limitan a aplicar las operaciones aritméticas, sino que también requieren de la aplicación de conocimientos no matemáticos o que contienen datos irrelevantes.

El objetivo general de este artículo es aportar algunas claves para comprender estas dificultades y para ayudar a los alumnos a superarlas. Para ello en primer lugar, presentaremos el Informe PISA como indicador de las dificultades que muestran los alumnos al resolver problemas que describen situaciones de la vida real. En segundo lugar, recogemos los resultados de varios estudios en los que constata cómo los alumnos encuentran dificultades persistentes en la resolución de problemas que se asemejan a los ítems de evaluación del informe PISA, los problemas realistas, e interpretaremos los resultados de esos estudios. En tercer lugar, describiremos las posibles razones por las cuales los niños muestran esas dificultades para resolver correctamente este tipo de problemas. Finalmente, proponemos una serie de orientaciones para los docentes que pueden contribuir a mejorar el rendimiento de los alumnos en la resolución de estos problemas.

\section{Los “problemas” del informe PISA}

El carácter instrumental de las matemáticas hace que de manera periódica salte la alarma a propósito de los resultados que los alumnos de muchos países (entre ellos el nuestro) obtienen en el área de matemáticas de ciertas evaluaciones internacionales como el informe PISA, especialmente en el apartado de resolución de problemas. Probablemente, una de las causas de este bajo rendimiento esté en qué se entiende por competencia matemática y qué tipo de problemas se utilizan para evaluarla. El informe define la competencia matemática como "la aptitud de un individuo para identificar y comprender el papel que desempeñan las matemáticas en el mundo, alcanzar razonamientos bien fundados y utilizar y participar en las matemáticas en función de las necesidades de su vida como ciudadano constructivo, comprometido y reflexivo" (INECSE, 2004).

Un ejemplo de problema que evalúa la competencia matemática entendida de este modo, tomado de los ejemplos de ítems recogidos por el Instituto Vasco de Evaluación e Investigación Educativa (2005, pp. 76-77), es el siguiente:

Isaac, de 15 años, quiere organizar una salida al cine con dos amigos de su misma edad durante la semana de vacaciones escolares. Las vacaciones empiezan el sábado, 24 de marzo, y terminan el domingo, 1 de abril.

Isaac preguntó a sus amigos qué días y a qué horas podrían ir al cine. Recibió las siguientes respuestas.

Federico: Tengo que quedarme en casa el lunes y el miércoles para practicar música de 14:30 a 15:30

Sebastián: Tengo que ir a casa de mi abuela los domingos, de modo que no puede ser en domingo. Ya he visto Pokamin y no quiero verla otra vez. 
Los padres de Isaac insisten en que sólo vaya a ver películas recomendadas para su edad y en que no vuelva a casa andando. Ellos llevarán a los chicos a sus casas siempre que sea antes de las 22 horas. Isaac mira las horas de comienzo de las películas de la semana de vacaciones. Ésta es la información que encuentra.

\begin{tabular}{|c|c|c|c|}
\hline Pelia & $\begin{array}{r}\text { Cl } \\
\text { Reserva anticipa } \\
\text { Teléfono } 2 \\
\text { Martes, dia del esp } \\
\text { las que se exhiben a } \\
\text { permanecerán }\end{array}$ & $\begin{array}{l}\text { riVOLI } \\
\text { entradas: } 924576425 \\
\text { r: } 9245766303 \\
\text { dodas las peliculas a } \\
\text { del Viernes } 23 \text { de ma } \\
\text { ntalla dos semanas. }\end{array}$ & 3 \\
\hline $\begin{array}{l}\text { Los Ninios en la Red } \\
113 \text { mimeos } \\
14: 00 \text { (solo Lun a Ve.) } \\
21: 35 \text { (s010 Sab. y Dom) }\end{array}$ & $\begin{array}{l}\text { No recomendada pasa } \\
\text { menores de } 12 \text { anos. }\end{array}$ & $\begin{array}{l}\text { Pokamin } \\
105 \text { minutos } \\
13: 40 \text { (a diario) } \\
\text { 16:35 (a diatio) }\end{array}$ & $\begin{array}{l}\text { Con autorizacion do los pades } \\
\text { Para todos los publicos, pero } \\
\text { algunas escenas pueden no } \\
\text { ser adecuades para los mass } \\
\text { jovenes. }\end{array}$ \\
\hline $\begin{array}{l}\text { Monstruos en las profur } \\
164 \text { minutos } \\
19.55 \text { (solo Ve. a Sab.) }\end{array}$ & $\begin{array}{l}\text { didades } \\
\text { No recomendada para } \\
\text { menores de } 18 \text { anos. }\end{array}$ & $\begin{array}{l}\text { Enigma } \\
\text { 144 minutos } \\
\text { 15:00 (solo lun. a Vie.) } \\
\text { 18:00 (sollo Sab. y Dom) }\end{array}$ & $\begin{array}{l}\text { No recomendada para } \\
\text { menores de } 12 \text { anch. }\end{array}$ \\
\hline $\begin{array}{l}\text { Carnivoro } \\
148 \text { minutos } \\
18: 30 \text { (a diario) }\end{array}$ & $\begin{array}{l}\text { No recomendada para } \\
\text { menores de } 18 \text { anos. }\end{array}$ & $\begin{array}{l}\text { E1 Rey de la Selva } \\
117 \text { minutos } \\
\text { 14:35 (sclo Lun. a Vie.) } \\
\text { 18:50 (solo Sab. y Dom.) }\end{array}$ & Para todos los públicos. \\
\hline
\end{tabular}

Teniendo en cuenta la información que ha encontrado Isaac sobre las películas y las condiciones que le ponen sus amigos, ¿cuál o cuáles de las seis películas son las que podrían ir a ver Isaac y sus compañeros?

Como puede observarse, el problema que hemos tomado como ejemplo requiere que los alumnos hagan uso de su conocimiento matemático (por ejemplo, para determinar qué hora acaba cada película) pero también que atiendan a información no matemática y que discriminen aquella información que es relevante para la tarea de la que no lo es (por ejemplo, es relevante el tipo de película o el día de emisión, pero no que el día del espectador sea el martes).

Los resultados alcanzados tras la aplicación de este ítem a los alumnos españoles fueron que menos de la mitad de los alumnos resolvió de manera satisfactoria el problema. En el caso del test completo, los resultados no fueron más alentadores: en el área de matemáticas los alumnos españoles obtuvieron una puntuación inferior a la de la media de la OCDE, especialmente en los ítems de resolución de problemas. Así, de los resultados de informes como el PISA podemos concluir que a los alumnos les cuesta resolver problemas relacionados con situaciones que se asemejan a las de la vida real. Sin embargo, cabría preguntarse hasta qué punto los resultados de este informe reflejan la realidad de las aulas, esto es, si los alumnos realmente tienen las dificultades en resolución de problemas que los resultados del Informe reflejan. En el siguiente epígrafe trataremos de responder a esta pregunta.

\section{Un problema real: cuando los alumnos sólo calculan}

Respondiendo a la pregunta que acabamos de plantear sobre si realmente los alumnos tienen tantas dificultades al resolver problemas que requieren de la aplicación de conocimientos más allá de los matemáticos, como se desprende de los resultados del Informe, estas dificultades han sido ampliamente documentadas en el ámbito de la resolución de problemas realistas, esto es, en problemas que reproducen situaciones problemáticas presentes en la vida cotidiana y en el trabajo (p.e.: Burkhardt, 1994) y para cuya resolución es necesario saber cuándo y cómo debe aplicarse el conocimiento matemático, pero también no matemático (Verschaffel, Greer y De Corte, 2000). Como puede observarse, esta definición se aproxima a la aportada por el informe PISA sobre la necesidad de fundamentar 
coherentemente los razonamientos subyacentes a la resolución de problemas y sobre la necesidad de aprender a resolver problemas de la vida real.

Los estudios que mejor han documentado las dificultades de los niños cuando se enfrentan a la resolución de problemas realistas han sido los desarrollados por Greer (1993), en Irlanda del Norte y Verschaffel, De Corte y Lasure (1994) en Bélgica. Estos autores comprobaron que los estudiantes de Educación Secundaria, en el caso de Greer, y de alumnos de $5^{\circ}$ de Educación Primaria en el caso de Verschaffel et al. no aplican razonamientos sobre la realidad que, sin ser matemáticos, son relevantes para resolver el problema. Ambos estudios emplearon la misma metodología: en el transcurso de una sesión normal de matemáticas se les administró a los alumnos una prueba experimental de lápiz y papel en la que se presentaron pares de ítems. En cada par, el primer item era un problema estándar que podía resolverse aplicando únicamente operaciones aritméticas (p.e.: "Un hombre corta una tela de 12 metros en piezas de 1.5 metros cada una. ¿Cuántas piezas de tela obtendrá?"). El segundo ítem de cada par era "problemático" en el sentido de que necesitaba de la realización de consideraciones sobre el mundo real (p.e.: "Un hombre quiere obtener una cuerda lo suficientemente larga como para atar dos postes que distan 12 metros entre sí, pero sólo tiene trozos de cuerda de 1.5 metros de largo. ¿Cuántas de esas piezas necesitará para atar los dos postes?")

Los 10 ítems problemáticos utilizados por Verschaffel et al. (1994) son los que figuran en la tabla I.

TABLA I

Ítems problemáticos utilizados por Verschaffel et al. (1994)

Carlos tiene 9 amigos y Jorge tiene 12 amigos. Carlos y Jorge deciden dar juntos una fiesta. Invitan a todos sus amigos, y todos los amigos van a la fiesta.¿Cuántos amigos van a la fiesta?

Esteban ha comprado 4 tablones de 2.5 metros cada uno ¿Cuántos tablones de 1 metro puede obtener de esos tablones?

¿Cuál será la temperatura del agua de un recipiente si mezclas un litro de agua a $80^{\circ}$ y un litro de agua a $40^{\circ}$ en él?

450 soldados deben ser transportados a su lugar de entrenamiento. En cada autobús pueden entrar 36 soldados.¿Cuántos autobuses serán necesarios?

Juan corre los 100 metros en 17 segundos. ¿Cuánto tardará en correr 1 kilómetro?

Roberto y Alicia van a la misma escuela. Roberto vive a 17 kilómetros de la escuela y Alicia a 8 km. ¿A qué distancia vive Roberto de Alicia?

El abuelo da a sus 4 nietos una caja con 18 globos para repartir entre ellos. ¿Cuántos globos le toca a cada uno?

Andrés nació en 1978. Ahora estamos en 1993. ¿Cuántos años tiene?

Un hombre quiere tener una cuerda los suficientemente larga para unir dos postes separados entre si 12 metros, pero solo tiene trozos de cuerda de 1,5 metros. ¿Cuántos trozos necesitaría juntar para hacer la cuerda lo suficientemente larga para unir las estacas?

Este recipiente se está llenando con un grifo a un ritmo constante. Si el agua tiene una profundidad de $4 \mathrm{~cm}$ tras 10 segundos, ¿cuánta profundidad tendrá después de 30 segundos? (este problema se acompaña por el dibujo de un recipiente de forma cónica) 
Las respuestas aportadas por los alumnos a estos problemas se categorizaron en dos grupos. En primer lugar, se catalogaron como respuestas "realistas" aquellas respuestas que consideraban explícitamente el contexto del problema, y aquellas que aún sin hacer tales considerariones incluían comentarios realistas. A este segundo tipo de respuestas realistas los autores las denominaron "reacciones realistas" (RR). En segundo lugar, se consideraron respuestas "no realistas" aquellas que no mostraban ningún indicio de activación y uso del conocimiento sobre el mundo real.

Por ejemplo, a partir del problema de la cuerda, una respuesta realista sería "más de 8 piezas," pero también la respuesta " 12 : 1.5 = 8;" pero con un comentario añadido del tipo "no serán suficientes porque hay que atar las piezas. Una respuesta no realista sería “ $12: 1.5=8$ ” sin dar ninguna explicación adicional.

Tanto en el estudio de Greer (1993) como en el de Verschaffel et al. (1994) los alumnos tendían claramente a no realizar consideraciones realistas al resolver el ítem problemático de cada par. De todos los tipos de ítems problemáticos sólo en dos los alumnos dieron algunas respuestas con consideraciones realistas: el item de los autobuses ( 4 en la Tabla I) y el de los globos ( 7 en la Tabla I), que elicitaron un $49 \%$ y un $59 \%$ de respuestas realistas respectivamente.

Resultados similares a los obtenidos por estos dos estudios han sido documentados en varios países como Alemania (Renkl, 1999), Japón (Yoshida, Verschaffel y De Corte, 1997), Irlanda del Norte (Caldwell, 1995), Suiza (Reusser y Stebler, 1997b) y Venezuela (Hidalgo, 1997). En algunos de esos estudios (p.e.: Caldwell, 1995; Hidalgo, 1997) los investigadores además indagaron, mediante una entrevista individual con los alumnos, en las razones por las cuales no aplicaron el conocimiento del mundo real para adaptar sus respuestas a las restricciones impuestas por el contexto. A pesar de que estas entrevistas únicamente proporcionaron evidencias anecdóticas al respecto, permitieron constatar que al menos una parte de los alumnos creían explícitamente que una cosa es el mundo (artificial) de las matemáticas aplicadas a la resolución de problemas en la escuela, y otra muy diferente el mundo real fuera de la escuela. Nos detendremos en esta idea con detalle más adelante.

Cabría pensar que estos resultados se debieran al contexto experimental en el que se evaluó a los alumnos, y no a una creencia real en la separación dicotómica de lo matemático versus lo real. Más concretamente, cabe la posibilidad, en primer lugar, de que los resultados se debieran a la ausencia, en las instrucciones que acompañaban a la prueba experimental, de una advertencia sobre el carácter no estándar del test y de una invitación explícita para dar respuestas alternativas a los problemas. Esto es, los ítems problemáticos de los estudios descritos estaban formulados en términos similares a los problemas estándar, y además aparecían mezclados con éstos, de manera que es posible que, sin una advertencia explícita, los alumnos no llegaran a percatarse de las diferencias entre unos y otros. En segundo lugar, es posible también que el contexto de resolución de ítems problemáticos, idéntico al de los no problemáticos, les llevara a resolverlos siguiendo procedimientos similares.

Para comprobar ambas posibilidades se desarrollaron varios estudios en los que, por un lado, se proporcionaba a los alumnos indicaciones explícitas de que algunos de los problemas requerían de un análisis minucioso para ser resueltos, o bien se les daba directa y explícitamente ayuda para considerar diferentes respuestas alternativas teniendo en cuenta las realidades del contexto del problema (p.e.: Reusser y Stebler, 1997a; van Lieshout, Verdwaald y van Herk, 1997; Verschaffel, De Corte y Lasure, 1999; Yoshida et al, 1997), y por otro se incrementaba la autenticidad de las condiciones experimentales (DeFranco y Curcio, 1997; Reusser y Stebler, 1997b; Wyndhamn y Säljö, 1997) 
Un ejemplo del primer conjunto de estudios es el realizado por Yoshida et al. (1997). Los autores compararon el rendimiento en resolución de problemas realistas de una muestra de alumnos de $5^{\circ}$, dividida en dos grupos. Al primer grupo se le administró el test de la misma manera que en el estudio de Verschaffel et al. (1994), mientras que a los alumnos del segundo grupo se les proporcionó información adicional escrita en la parte superior de la hoja del test, indicando explícitamente la existencia de problemas difíciles o imposibles de resolver cuyo enunciado contenía aspectos poco claros o complejos. Los resultados mostraron un ligero aumento que no alcanzó la significatividad a favor del grupo que recibió esas instrucciones. Los porcentajes globales de respuestas realistas fueron del $15 \%$ y del $20 \%$ para el primer y el segundo grupo, respectivamente. El resto de estudios similares al de Yoshida et al. (1997) obtuvieron resultados semejantes, de modo que podemos concluir que las modificaciones en las condiciones experimentales introducidas para alertar a los alumnos sobre el carácter especial de los ítems problemáticos únicamente produjeron efectos muy débiles.

En el segundo conjunto de estudios se incrementó la autenticidad de las condiciones experimentales presentando una o más categorías de ítems problemáticos en unas condiciones más auténticas, esto es, en el contexto de un grupo de discusión y/o con la ayuda de ayudas concretas. Por ejemplo, DeFranco y Curcio (1997) tomaron problemas de división con resto, y propusieron dos configuraciones experimentales diferentes: una configuración escolar restrictiva, que consistía en una entrevista individual en la que se preguntaba a los alumnos sobre la resolución matemática del problema, y otra (relativamente) realista, en la que se pedía a los alumnos que realizaran una llamada telefónica para reservar autocares para llevar a todos los alumnos de $6^{\circ}$ curso a una fiesta. El resultado fue que 18 de los 20 niños respondieron de manera inadecuada al problema de los autobuses en el contexto experimental restrictivo, de los cuales 17 realizaron una interpretación incorrecta del resto (aportando resultados con resto o redondeando a la baja hasta 8 autobuses). En contraste, en la configuración experimental realista 16 de los 20 alumnos dieron una respuesta apropiada.

En definitiva, este segundo conjunto de estudios (DeFranco y Curcio, 1997; Reusser y Stebler, 1997b; Wyndhamn y Säljö, 1997), que proponían contextos de resolución más realistas, produjeron una enorme mejora en el rendimiento de los alumnos en los ítems problemáticos. En concreto, los alumnos mostraron una mayor capacidad para utilizar su conocimiento sobre el mundo real para realizar consideraciones realistas.

Los resultados de los trabajos que acabamos de describir indican que las dificultades que mostraron los alumnos a la hora de resolver los problemas del Informe PISA reflejan una realidad documentada por otros estudios en el ámbito de la resolución de problemas realistas, semejantes a los utilizados por ese Informe. Además, hemos comprobado que estas dificultades persisten aún cuando se advierte a los alumnos del carácter "especial" de los problemas realistas, pero se reducen cuando se les pide que resuelvan los problemas en contextos experimentales más cercanos a la vida real que a la vida escolar. Esto nos lleva a pensar que algún factor de esa vida escolar les impide resolver correctamente este tipo de problemas. En el siguiente punto trataremos de perfilar ese factor

\section{Razones para la inhibición del conocimiento sobre el mundo real en la resolución de problemas realistas}

Tal y como acabamos de describir, diversos estudios han demostrado que los alumnos raramente apelan a razonamientos no matemáticos para resolver problemas de matemáticas, ni siquiera cuando se les advierte de que los pro- 
blemas a resolver probablemente requieran considerar elementos no matemáticos para su resolución. Sólo cuando se les introduce en un contexto más real son capaces de adaptarse a él y de aplicar razonamientos no estrictamente matemáticos. En otras palabras, estos resultados sugieren que cuando la naturaleza de las "premisas para el ritual interactivo" (Wyndhamn y Säljö, 1997, p. 379) o lo que, de manera más general, Greer (1997, p. 305) ha denominado, el "contrato experimental” lo permite, los alumnos se muestran competentes y son capaces de hacer consideraciones realistas cuando resuelven un problema matemático.

De esta manera, podemos señalar que el factor subyacente a la resistencia de los alumnos a considerar elementos ajenos al razonamiento matemático para resolver los problemas realistas es el "contrato experimental" que acabamos de referir en palabras de Greer (1997). Este contrato establece que: a) la resolución consiste simplemente en elegir y ejecutar una (o varias) de las cuatro operaciones básicas; b) estas operaciones deben aplicarse sobre (todos) los números que aparecen en el enunciado del problema, y; c) no es necesario considerar seriamente las posibles incoherencias de la aplicación de estas operaciones respecto al mundo real al que alude la situación descrita por el problema, comprometiendo de esta manera el ajuste de sus modelos y de las soluciones propuestas (Davis, 1989; Greer, 1997; Freudental, 1991; Kilpatrick, 1987; Nesher, 1980; Reusser, 1988; Schoenfeld, 1991). Más concretamente, varios autores han analizado las reglas y presupuestos "ocultos" que gobiernan (implícita o tácitamente) las interacciones maestros-alumnos cuando se enfrentan a la resolución de problemas (De Corte y Verschaffel, 1985; Gerofsky, 1996; Lave, 1992; Reusser y Stebler, 1997a; Schoenfeld, 1991). Estos presupuestos son los siguientes:

- Todo problema presentado por el maestro o por el libro de texto puede resolverse y tiene sentido.

- Cada problema tiene una única respuesta correcta, y ésta es precisa y numérica.

- La solución de cada problema puede y debe obtenerse ejecutando una o más operaciones aritméticas con los números del problema, y casi con toda seguridad con todos ellos.

- La tarea puede realizarse con las matemáticas que han aprendido como estudiantes, en la mayoría de los casos aplicando los conceptos, fórmulas, y algoritmos matemáticos expuestos en las clases más recientes

- La solución final, e incluso el resultado intermedio, implica números "limpios" (generalmente números enteros pequeños)

- El problema por sí mismo contiene toda la información necesaria para generar la interpretación matemática correcta y llegar a la solución del problema, de modo que no debe buscarse información extraña.

- Finalmente, las personas, objetos, lugares y razonamientos son diferentes en los problemas de matemáticas de la escuela que en las situaciones del mundo real, por lo que no hay que preocuparse demasiado si la situación propuesta por el problema viola los conocimientos previos o las intuiciones basadas en las experiencias cotidianas.

¿Cuáles podrían ser las causas para el establecimiento de este "contrato"? Varios autores (De Corte y Verschaffel, 1985; Gerofsky, 1996; Kilpatrick, 1987; Lave, 1992; Reusser y Stebler, 1997a; Schoenfeld, 1991) han señalado que las creencias en las que se sustenta ese "contrato" se desarrollan de manera gradual y tácita a través de la inmersión en la cultura de la matemática del aula. A su vez, esta enculturación parece deberse principalmente a dos aspectos de la práctica instruccional: por un lado, qué resuelven los 
alumnos, esto es, la naturaleza de los problemas de matemáticas que resuelven, y por otro cómo lo resuelven, esto es, el tipo de interacciones y actividades que se generan en torno a estos problemas. En las siguientes páginas nos detendremos en cada uno de ellos.

\section{Qué resuelven los alumnos: problemas de matemáticas en los libros de texto}

Tal y como afirman Orrantia, González y Vicente (2005), buena parte del tiempo que los alumnos dedican a resolver problemas de matemáticas lo hacen utilizando materiales preparados para tal fin, de entre los cuales el libro de texto tiene un papel predominante. Muchos estudios han documentado cómo los libros de texto influyen claramente en las prácticas educativas de los profesores (Cooney, 1985; Haggarty y Pepin, 2002; Millet y Johnson, 1999; NCTM, 1989; Schimtz, McNight, Valverde, Houang y Wiley, 1997; Stray, 1994). De hecho, los profesores suelen partir de los libros de texto, de su estructura y de la visión que aportan sobre qué es resolver problemas y su función dentro del currículum en matemáticas (Nathan y Koedinger, 2000), de manera que la influencia del libro de texto en cómo se enseña a los alumnos a resolver problemas puede ser muy profunda.

De los numerosos estudios internacionales que han analizado los libros de texto (Carter, Li y Ferrucci, 1997; De Corte, Verschaffel, Janssens y Joillet, 1985; Fuson, Stigler y Bartsch, 1988; Li, 2000; Mayer, Sims y Tajika, 1995; Orrantia et al., 2005; Stigler, Fuson, Ham y Kim, 1986) pueden extraerse dos conclusiones. La primera de ellas es que los libros presentan y agrupan los problemas de manera que para resolver la mayoría de ellos los alumnos únicamente han de aplicar estrategias superficiales (utilizando para ello "palabras clave", como "más" o "ganar" para sumar) o simplemente aplicar la operación cuyo aprendizaje sea el objetivo de ese momento en concreto (en el caso de que sea restar, probablemente todos los problemas de esa lección se resuelvan restando). La segunda conclusión a la que permiten llegar los resultados de esos estudios es que los problemas desafiantes, esto es, aquellos que contienen información innecesaria o los que omiten deliberadamente uno de los datos necesarios para resolver el problema, son poco frecuentes en los textos, reforzando así el carácter estereotipado de la tarea de resolución de problemas en las clases de matemáticas.

Resumiendo las principales características de los problemas tradicionales, Reusser y Stebler (1997a, p. 323) afirmaban que:

Sólo unos pocos problemas empleados en las aulas y en los libros de matemáticas invitan o desafían a activar y utilizar su conocimiento sobre el mundo real y su experiencia. La mayoría de problemas utilizados en la instrucción se elaboran de manera semánticamente empobrecida, a modo de viñetas verbales. Los alumnos no sólo saben por su experiencia matemática escolar que todos los problemas son indudablemente resolubles, sino que también saben que cualquier cosa numérica incluida en un problema es relevante para su resolución, y que todo lo que es relevante para su resolución está incluido en el texto del problema. Siguiendo este código, los enunciados de muchos problemas degeneran en ecuaciones mal disimuladas.

De esta manera, los alumnos terminan por deducir que cuando se enfrentan a la resolución de un problema lo que se les pide es que ejecuten una o varias operaciones aritméticas con todos los números que aparecen en el mismo. Igualmente, las pocas ocasiones en las que aparece información adicional no numérica en el enunciado del problema con frecuencia es información no relevante para la comprensión del mismo, reduciéndose la presentación de información contextual a unas pocas situaciones estereotipadas (Orrantia et al., 2005). 
Cómo se resuelven los problemas en el aula: creencias de los profesores e interacciones profesor-alumno

Como acabamos de señalar, la mayor parte de los problemas de matemáticas que aparecen en los libros de texto -que son en definitiva, los que se resuelven en las aulas- favorecen la resolución mediante procedimientos exclusivamente matemáticos y, en la mayoría de las ocasiones, sin necesidad de una comprensión profunda del problema. El segundo elemento que contribuye al establecimiento del "contrato" de aula que fija las reglas del proceso de resolución de problemas en la escuela es el conjunto de interacciones que mantienen maestros y alumnos para resolver esos problemas.

Evidentemente, quien aporta las pautas para establecer esta interacción es el maestro. De esta manera, las creencias que alberga el maestro sobre qué es un problema de matemáticas y cómo debe ser resuelto influyen de manera determinante en el rumbo que tomarán las interacciones con sus alumnos. En este sentido, Verschaffel, De Corte y Borghart (1997) realizaron un estudio muy interesante sobre las creencias que futuros maestros de Educación Primaria tienen sobre el rol del conocimiento del mundo real en el proceso de resolución de problemas en las clases de matemáticas. Los autores tomaron una muestra de 332 maestros de Educación Primaria en formación de tres centros de formación diferentes de Flandes (Bélgica). Una parte de los sujetos acababa de comenzar su primer año de formación, mientras que el resto eran alumnos de tercer año que prácticamente habían completado su formación como maestros. La tarea a la que se sometió a los sujetos consistía en una prueba experimental que constaba de 14 problemas: siete ítems estándar no problemáticos y siete ítems problemáticos seleccionados de entre los utilizados en los estudios previos.

Se realizaron dos aplicaciones de la tarea experimental a todos los sujetos del estudio. La primera aplicación (test 1) debían responder los 14 problemas por sí mismos, añadiendo a los cálculos cualquier comentario sobre el problema en un espacio habilitado para tal fin en la hoja de respuestas. Inmediatamente después de terminar la primera aplicación se realizaba la segunda, en la que debían puntuar cuatro respuestas alternativas, típicas de los niños a los problemas de la tarea experimental, con 1 punto (respuesta absolutamente correcta), 0 (respuesta absolutamente incorrecta) o $1 / 2$ punto (respuesta parcialmente correcta y parcialmente incorrecta). Las cuatro posibles respuestas de los alumnos que los futuros profesores tenían que valorar podían pertenecer a cuatro categorías, que se ilustran en la figura 1: una respuesta no realista (opción A), una respuesta realista (opción D), un error en la operación (opción B) y, finalmente, cualquier otra respuesta (opción C).

Para analizar los resultados se clasificaron las respuestas espontáneas de los maestros en formación a los ítems problemáticos en dos categorías: reacciones realistas y reacciones no realistas. El análisis de las reacciones de estos maestros a los siete ítems problemáticos del segundo test se centraron en la puntuación (1, $1 / 2$ o 0 ) dadas a las respuestas realistas y a las respuestas no realistas.

Ambas aplicaciones mostraron tres resultados muy claros. En primer lugar, los futuros maestros mostraron una fuerte tendencia no utilizar el conocimiento del mundo real y a no realizar consideraciones realistas ni al resolver los ítems problemáticos del test $1 \mathrm{ni}$ al valorar las respuestas de los alumnos en el test 2 . En segundo lugar, los sujetos que se encontraban en su tercer año de formación mostraron una proporción significativamente mayor de respuestas realistas que los de primer año en ambos tests. Finalmente, la diferencia de porcentajes de RRs en los ítems problemáticos entre los sujetos de primer y tercer año variaba notablemente entre los tres centros de forma- 
FIGURA 1

Presentación de los problemas en el test 2 (Verschaffel et al., 1997)

Este frasco se está llenando con un grifo a un ritmo constante. Si la profundidad del agua es de $3.5 \mathrm{~cm}$. después de 10 segundos, ¿cuál será la profundidad después de 30 segundos?

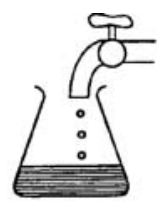

$3 \times 3.5 \mathrm{~cm} .=11.5 \mathrm{~cm}$.

Después de 30 segundos la profundidad del agua será de $11.5 \mathrm{~cm}$.

A

$3.5 \mathrm{~cm} .+20 \mathrm{~cm} .=23.5 \mathrm{~cm}$.

Después de 30 segundos la profundidad del agua será de $11.5 \mathrm{~cm}$.
$3 \times 3.5 \mathrm{~cm} .=10.5$.

Después de 30 segundos la profundidad del agua será de $10.5 \mathrm{~cm}$.

B

Es imposible dar una respuesta precisa

$\mathrm{C}$

$\mathrm{D}$

ción que participaron en el estudio, lo cual indica que la disposición para resolver los problemas el modelado realista de los problemas mostrado por los futuros maestros estaba influenciada, al menos parcialmente, por la formación en educación matemática recibida durante los cursos de formación. De estos resultados se desprende que muchos futuros maestros albergan conocimientos y creencias sobre cómo debe enseñarse y cómo deben aprender los alumnos a resolver problemas que dificultan la aplicación de conocimientos sobre el mundo real y la realización de consideraciones realistas.

Sin embargo, es necesario señalar que aún no hay evidencias directas de que estas concepciones y creencias de los profesores sean la causa de la fuerte tendencia de los alumnos a excluir el conocimiento del mundo real de sus procedimientos de resolución de problemas. Además, queremos destacar que no es de ninguna manera nuestra intención reprochar a estos futuros maestros sus comportamientos ni sus creencias. Al igual que los alumnos, estos futuros maestros en el estudio de Verschaffel et al. (1997) estaban actuando (algunas veces de manera deliberada, pero en la mayoría de las ocasiones de manera inconsciente) de manera "racional" de acuerdo con su experiencia sobre el contrato didáctico que rige la resolución de problemas.

\section{Conclusión: un proceso de resolución basado en una comprensión limitada}

Como hemos argumentado hasta ahora, el bajo rendimiento de los alumnos a la hora de resolver problemas realistas, similares a los utilizados en informes internacionales de Evaluación como el PISA probablemente se deba a una cultura determinada tanto por el tipo de problemas que se resuelvan en el aula como por el modo de resolverlos en el aula. Asimismo, las creencias de los profesores sobre cómo deben ser resueltos los problemas podría determinar el rumbo que toma la interacción con los alumnos cuando los resuelven conjuntamente.

Por otra parte, cabe destacar que si bien los estudios que han constatado de manera empírica los efectos de la cultura de aula sobre el comportamiento de los 
niños han sido escasos -excepto en el caso en el que los alumnos que se enfrentaban a problemas del tipo “Cuántos años tiene el capitán?" (IREM de Grenoble, 1980) o el problema de los autobuses (problema realista del tipo división con resto) en el National Assessment of Educational Progress (NAEP) en los Estados Unidos (Carpenter, Lindquist, Matthews y Silver, 1983)-, los estudios más recientes indican que muchos alumnos resuelven los problemas de acuerdo con una comprensión limitada de los problemas. Esta limitación en la comprensión del problema se debe a que utilizan el texto del problema únicamente para elegir una (o más) de las cuatro operaciones aritméticas de dos formas diferentes. En primer lugar, pueden utilizar los rasgos superficiales del problema, tales como determinadas palabras clave en el texto (por ejemplo, la palabra "menos" en el texto automáticamente guía la elección de la resta como operación a realizar para resolver el problema). En segundo lugar, pueden establecer una asociación entre la situación descrita en el problema y un modelo rudimentario para una de las operaciones (por ejemplo, cualquier cosa que sugiera el acto de "juntar" indica que hay que sumar).

Una vez determinada la operación a realizar, los alumnos la aplican automáticamente a los números que aparecen en el enunciado, y la respuesta al mismo es el resultado obtenido de la aplicación de la operación sugerida por la palabra clave sobre los números del enunciado, sin volver al enunciado del texto para verificar que la respuesta tiene sentido considerando la pregunta del problema.

\section{En busca de soluciones: propuestas para la resolución de problemas realistas}

Muchos estudios experimentales han dado cuenta de cómo los alumnos acometen la resolución de problemas realistas de una manera altamente estereotipa$\mathrm{da}$, probablemente como consecuencia de un proceso de aprendizaje marcado por una cultura de aula que no promueve el uso de conocimientos no matemáticos en la resolución de problemas. Ante este panorama, cabe preguntarse qué puede hacerse para mejorar el rendimiento de los alumnos en este tipo de problemas.

Una respuesta podemos encontrarla en los estudios instruccionales que han tratado de de mejorar el rendimiento de los alumnos cuando se enfrentan a este tipo de problemas modificando el proceso de enseñanza-aprendizaje de resolución de problemas realistas. Como ejemplo de estos estudios instruccionales tomaremos el desarrollado por Verschaffel y De Corte (1997b). Estos autores diseñaron un experimento de enseñanza a pequeña escala cuyos objetivos eran dos: por un lado, modificar las creencias de los alumnos acerca de la función del conocimiento sobre el mundo real en el proceso de resolución de problemas realistas, y por otro desarrollar en los alumnos la capacidad de resolver los problemas siguiendo pautas más realistas. Para ello modificaron la cultura del aula en la que los alumnos estaban inmersos de manera que los problemas se concibieran como oportunidades de aplicar sus conocimientos, tanto los matemáticos como los relativos al mundo real. El estudio incluyó tres clases de los últimos cursos de Educación Primaria de Flandes (Bélgica), la primera experimental y otras dos de control. Los alumnos de la clase experimental formaron parte de un programa de instrucción sobre resolución de problemas realistas, que constaba de cinco unidades de dos horas y media cada una, mientras que los alumnos de las clases de control siguieron las clases correspondientes a su currículum ordinario. Las principales características del programa instruccional al que fue sometido el grupo experimental eran tres. En primer lugar, se sustituyeron los problemas estereotipados típicos de las clases habituales por problemas realistas, que incitaban a los 
alumnos a atender a las complejidades de estos problemas. En segundo lugar, las unidades se basaron en el trabajo colaborativo en pequeño grupo, seguido por discusiones de toda la clase. Finalmente, se trató de establecer una nueva cultura de aula a través de la negociación explícita de nuevas normas sociales relativas tanto a los roles del profesor y de los alumnos como a qué se entiende por problema de matemáticas, por resolver correctamente un problema y por qué se considera una buena respuesta al problema (ver Cobb, Wood y Yackel, 1993; Schoenfeld, 1991).

Los alumnos de las tres clases fueron sometidos a un pre-test y a un post-test que incluía ítems de aprendizaje, de retención y de transferencia. Los resultados mostraron que, partiendo de un nivel similar de rendimiento en resolución de problemas realistas, tras el estudio instruccional los alumnos del grupo experimental rindieron significativamente mejor en los tres tipos de ítems del posttest. De esta manera, los autores concluyeron que es posible modificar las creencias sobre el rol del conocimiento sobre el mundo real en la resolución de problemas de los alumnos de los cursos superiores de la Educación Primaria, y que pueden aprender a resolver los problemas de manera (más) realista.

Renkl (1999) replicó este experimento en Alemania, con resultados igualmente prometedores. Otros estudios instruccionales, con una perspectiva más amplia y una mayor validez ecológica, han documentado cómo sumergiendo a los alumnos en un ambiente de aprendizaje modificado pueden desarrollar creencias sobre la resolución de problemas y estrategias de resolución acordes con esas creencias más apropiadas a la resolución de problemas realistas (Cognition and Technology Group at Vanderbilt, 1997; Lehrer y Schauble, 2000; Verschaffel et al., 1999).

\section{Reconceptualizando los problemas de matemáticas.}

Al comienzo de este trabajo presentábamos la definición de competencia matemática que PISA toma como referencia, en la que se señalaba su carácter pragmático para la vida cotidiana de los sujetos, en los que las situaciones problemáticas suelen incluir factores no matemáticos que deben ser considerados para su resolución. También hemos descrito cómo la cultura escolar no comparte esa concepción de matemática ni la definición de resolución de problemas que de ella se deriva, ya que en la escuela resolver problemas suele ser sinónimo de ejercitar operaciones prácticamente sin razonamiento. Finalmente, hemos planteado la idea de que a los resultados mediocres que los alumnos suelen obtener en este tipo de evaluaciones subyace la distancia entre ambas concepciones y entre los tipos de problemas y prácticas educativas que de ellas se derivan de ellas, y que esta distancia puede acortarse modificando las prácticas instruccionales y el concepto de resolución de problemas subyacente a ellas.

Para concluir este trabajo de revisión creemos conveniente sintetizar las principales aportaciones de los estudios que hemos revisado a modo de sugerencias para la reconceptualización del papel de la resolución de problemas realistas. De esta manera, la primera de las sugerencias que queremos plantear es la conveniencia de establecer como objetivo mínimo y asequible el de mejorar la calidad de los problemas cuya resolución se propone a los alumnos. Para ello será necesario el cumplimiento de una serie de premisas:

- Es conveniente plantear los problemas de manera que no fomenten en los alumnos la creencia de que cualquier problema de matemáticas puede resolverse simplemente sumando, restando, multiplicando o dividiendo, o combinando esas operaciones 
- Sería recomendable eliminar de los libros de texto aquellos problemas que permitan estrategias superficiales de resolución.

- Es necesario variar los tipos de problemas a los que se enfrentan los alumnos incluyendo problemas con datos superfluos y problemas a los que les falte algún dato que tengan que inferir. De esta manera los alumnos aprenderán, por un lado, que no todos los datos incluidos en el problema son necesarios para su resolución, y por otro, que en ocasiones es necesario encontrar los datos que no se proporcionan explícitamente en el enunciado del problema.

- Conviene evitar aquellos problemas en los que las cantidades no se corresponden con la vida real.

- Sería positivo presentar como legítimas y válidas respuestas a los problemas diferentes a las respuestas numéricas exactas (estimaciones, aproximaciones....)

- En necesario generar ocasiones para que los niños inventen sus propios problemas

Afortunadamente, estas recomendaciones están siendo incorporadas, al menos en algún grado, en las nuevas generaciones de libros de texto de muchos países, entre ellos el nuestro.

En segundo lugar, es necesario modificar no sólo los problemas que aparecen en los libros de texto, sino también la enseñanza tradicional de las matemáticas y la resolución de problemas. Esto implica abandonar la creencia de que los problemas son meros ejercicios de práctica de operaciones aritméticas, para entender que resolver un problema de matemáticas es una actividad colectiva en la que:

a. el conocimiento sobre el mundo real al que alude el problema no se elimina sino que se considera necesario en el momento inicial del proceso de resolución,

b. se establecen unas metas explícitas respecto a la situación (resolver la situación problemática y no sólo el problema de matemáticas), bien impuestas por el maestro o bien negociadas, que guían el proceso de resolución

c. el alumno dispone de una amplia variedad de recursos (incluyendo software informático específico) para resolver los problemas,

d. la interpretación del proceso de resolución implica la comparación de los procedimientos alternativos, $y$

e. la expresión del resultado y no se limita a informar al resto de los compañeros y al profesor (para más detalles ver Verschaffel et al., 2000).

Algunos países ya han comenzado a implementar, al menos en algún grado, esta manera alternativa de entender los problemas de matemáticas, si bien, tal y como señala Niss (2001), estas iniciativas aún son escasas y su influencia en la práctica cotidiana muy limitada. Este autor señala como causa probable de esta escasa incidencia la dificultad que implica para los maestros, tanto a nivel matemático como pedagógico y personal, este cambio de perspectiva. Por otra parte, la implementación de esta manera de conceptualizar la resolución de problemas requiere de la implicación no sólo de los maestros, sino también de los investigadores educativos, de las instituciones, los legisladores educativos, y las propias editoriales.

Por último, creemos conveniente realizar tres reflexiones finales. La primera de ellas es preguntarnos hasta dónde es sensato llevar la necesidad de realizar consideraciones realistas para la resolución de problemas. Estamos de acuerdo con Gravemeijer (1997) en que en la actualidad hay, y probablemente habrá siempre, diferencias entre resolver problemas dentro y fuera de la escuela. Sin embargo, no es menos cierto que sería conveniente acercar la concepción implícita que profesores y alumnos tienen de la matemática escolar a esa definición que PISA aporta sobre la competencia matemática. Asimismo, es razonable pen- 
sar que mientras no se produzca una aproximación entre ambas concepciones, seguiremos obteniendo resultados negativos en sucesivos informes de evaluación como el que hemos descrito en este trabajo.

Una segunda reflexión, relacionada con la anterior, es si la resolución realista de problemas debería sustituir a la enseñanza más tradicional de resolución de problemas. Nuestra propuesta a este respecto sería la de la coexistencia de ambos tipos de instrucción, unida a una dieta instruccional de los alumnos que alterne problemas tradicionales, que permiten crear vínculos sólidos entre las operaciones matemáticas y modelos de la situación prototípicos y sin complejidades situacionales sobre las que crear discusiones, con otros problemas que permiten relacionar situaciones del mundo real con las matemáticas. De esta manera, la realización de consideraciones realistas, al menos tal y como la hemos expuesto en este trabajo, estaría dirigida a los problemas realistas, mientras que los problemas más clásicos, como los problemas aritméticos, en los que los alumnos han de aplicar esquemas matemáticos para comprender y resolver ciertas categorías de problemas, requerirían de una enseñanza que se ajusta mejor a la tradicional.

La tercera reflexión es si aprender a resolver problemas de manera realista es realmente útil para los alumnos en su vida cotidiana. Durante los últimos años varios autores (p.e.: Mukhopadhay y Greer, 2001) se han mostrado partidarios de que todos los alumnos desarrollen esta capacidad porque les beneficia tanto a ellos como individuos, convitiéndoles en "pensadores críticos que puedan utilizar las matemáticas como una herramienta para analizar los asuntos sociales y políticos, y que este uso pueda reflejarse, incluyendo sus limitaciones" (Mukhopadhay y Greer, 2001), como a la sociedad en general. Esto es, la mejor manera de evitar que las matemáticas y la vida escolar sean dos cosas inconexas en la vida de los alumnos es que las entiendan como una herramienta eficaz para el análisis de cuestiones personales y de la sociedad, para lo cual han de aprender a ser sensibles a los contextos a los que se refieren los problemas y a la diversidad de perspectivas desde los que se pueden abordar. Las evidencias de los trabajos revisados nos hacen pensar que comenzar a aplicar la perspectiva del modelado en la educación matemática para todos los alumnos desde una edad muy temprana no sólo es importante, sino además factible.

\section{Notas}

${ }^{1}$ El problema es el siguiente: “En un barco viajaban 26 carneros y 10 cabras. ¿Cuál es la edad del capitán?” Los resultados indicaron que 78 de los 97 alumnos de 7 y 8 años a los que se pidió que resolvieran el problema respondieron " 36 "

\section{Referencias}

Burkhardt, H. (1994). Mathematical applications in school curriculum. En T. Husén \& T. N. Postlethwaite (Eds.), The international encyclopedia of education ( $2^{\mathrm{a}}$ ed.) (pp. 3621-3624). Oxford/Nueva York: Pergamon Press.

CALDWELL, L. (1995). Contextual considerations in the solution of children's multiplication and division word problems. Tesis de graduación no publicada. Queen's University, Belfast, Irlanda del Norte.

Carpenter, T. P., Lindquist, M. M., Matthews, W. \& Silver, E. A. (1983). Results of the third NAEP mathematics assessment: Secondary school. Mathematics Teacher, 76, 652-659.

CARTER, J., LI, Y. \& FerRUCCI, B. (1997). A comparison of how textbooks present integer addition and subtraction in China and the United States. Mathematics Educator, 2 (2), 197-209.

CobB, P., Wood, T. \& YACKel, E. (1993). Discourse, mathematical thinking, and classroom practice. En E. Forman, N. Minick \& A. Stone (Eds.), Contexts for learning: Social cultural dynamics in children's development (pp. 117-148). Nueva York: Springer.

COGNition AND TeChNOLOGy Group at VANDERBILt (1997). The Jasper project: Lessons in curriculum, instruction, assessment, and professional development. Mahwah, NJ: Lawrence Erlbaum Associates.

CoONEY, T. J. (1985). A begginning teacher's view of problem solving. Journal of Research in Mathematics, 13 (2), 87-104

Davis, R. B. (1989). The culture of mathematics and the culture of schools. Journal of Mathematical Behavior, 8, 143-160.

De CORTE, E. \& VersCHAFFel, L. (1985). Beginning first graders' initial representation of arithmetic word problems. Journal of Mathematical Behavior, 4, 3-21. 
De Corte, E., Verschaffel, L., Janssens, V. \& Joillet, L. (1985). Teaching word problems in the first grade: A confrontation of educational practice with results of recent research. En T. A. Romberg (Ed.), Using research in the professional life of mathematics teachers (pp. 186-195). Madison, WI: Center for Education Research, University of Wisconsin.

DeFranco, T. C. \& CURCIO, F. R. (1997). A division problem with a remainder embedded across two contexts: Children's solutions in restrictive versus real-world settings. Focus on Learning Problems in Mathematics, 19 (2), 58-72.

FusON, K. C., Stigler, J. W. \& BARTSCH, K, (1988). Grade Placement addition and subtraction topics in Japan, mainland China, the Soviet Union, Taiwan and the United States. Journal of Research in Mathematical Education, 19, $449-456$

Freudenthal, H. (1991). Revisiting mathematics education. Dordrecht: Kluwer.

GEROFSKY, S. (1996). A linguistic and narrative view of word problems in mathematics education. For the Learning of Mathematics, $16(2), 36-45$.

GraveMEIJER, K. (1997). Solving word problems: A case of modelling? Learning and Instruction, 7, 389-397.

Greer, B. (1993). The modeling perspective on wor(l)d problems. Journal of Mathematical Behavior, 12, 239-250.

GreER, B. (1997). Modelling reality in mathematics classrooms: The case of word problems. Learning and Instruction, 7, $293-$ 307.

Haggarty, L. \& Pepin, B. (2002): An investigation of mathematics textbooks and their use in English, French and German classrooms: who gets an opportunity to learn what? British Educational Research Journal, 28 (4), 567-590

Hidalgo, M. C. (1997). L'activation des connaissances à propos du monde réel dans la résolution de problèmes verbaux en arithmétique. Tesis Doctoral no publicada. Université Laval, Québec, Canada.

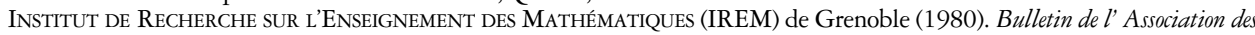
professeurs de Mathématique de l' Enseignement Public, 323, 235-243.

InStituto NaCiONAl DE Evaluación y CALIDAD DEl Sistema EdUCATIVo (INECSE) (2004). Aprender para el mundo de mañana. Resumen de resultados. PISA 2003. Madrid: Ministerio de Educación y Ciencia.

Instituto Vasco de Evaluación e InVestigación EduCativa (IVEI) (2005). Proyecto PISA 2003. Ejemplos de ítems de matemáticas y resolución de problemas. Bilbao: ISEI-IVEI.

Kilpatrick, J. (1987). Problem formulating: Where do good problems come from? En A. H. Schoenfeld (Ed.), Cognitive science and mathematics education (pp. 123-147) Hillsdale, NJ: Lawrence Erlbaum Associates.

Lave, J. (1992). Word problems: A microcosm of theories of learning. En P. Light \& G. Butterworth (Eds.), Context and cognition: Ways of learning and knowing (pp. 74-92). Nueva York: Harvester Wheatsheaf.

LeHreR, R. \& SCHAUble, L. (2000). Modeling in mathematics and science. En R. Glaser (Ed.), Advances in instructional psycho$\log$ (pp. 101-159). Mahwah, NJ: Lawrence Erlbaum Associates.

LI, Y., (2000). A comparison of problems that follow selected content presentations in american and chinese mathematics textbooks. Journal for Research in Mathematics Education, 31, 234-241.

MAYER, R. E., Sims, V. \& TAJIKA, H. (1995): Mathematical problem solving in Japan and the United States. American Educational Research Journal, 32, 443-460

Millett, A. \& Johnson, D. C. (1999). Odd one out? Some views of lay inspection. Cambridge Journal of Education, 29 (1), $63-$ 76.

Mukhopadhyay, S. \& Greer, B. (2001). Modelling with purpose. Mathematics as a critical tool. En B. Atweh, H. Forgasz \& B. Nebres (Eds.), Socio-cultural aspects in mathematics education (pp. 295-311). Mahwah, NJ: Lawrence Erlbaum Associates.

NATHAN, M. J. \& KOEDINGER, K. R. (2000). An investigation of teachers beliefs of students's álgebra development. Cognition and Instruction, 18 (2), 209-237.

National Council of Teachers of Mathematics (1989). Currículum and evaluation standard of school mathematics. Reston, VA: Autor.

NeSHER, P. (1980). The stereotyped nature of school word problems. For the Learning of Mathematics, 1 (1), 41-48.

Niss, M. (2001). Issues and problems of research on the teaching and learning of applications and modelling. En J. F. Matos, W. Blum, S. K. Houston \& S. P. Carreira (Eds.), Modelling and mathematics education. ICTMA 9: Applications in science and technology (pp. 72-89). Chichester, UK: Horwood.

Orrantia, J., GonZÁlez, L. B. \& Vicente, S. (2005). Un análisis de los problemas aritméticos en los libros de texto de Educación Primaria. Infancia Y Aprendizaje, 28(4), 429-451.

RENKL, A. (1999). The gap between school and everyday knowledge in mathematics. Conferencia presentada en la Eighth European Conference for Research on Learning and Instruction, Göteborg, Suecia, Agosto.

Reusser, K. (1988). Problem solving beyond the logic of things: Contextual effects on understanding and solving word problems. Instructional Science, 17, 309-338.

Reusser, K. \& STEBLER, R. (1997a). Every word problem has a solution: The suspension of reality and sense-making in the culture of school mathematics. Learning and Instruction, 7, 309-328.

REUSSER, K., \& STEBLER, R. (1997b). Realistic mathematical modeling through the solving of performance tasks. Comunicación presentada en la Seventh European Conference on Learning and Instruction, Atenas, Grecia, Agosto.

Schmidt, W. H., McKnight, C. C., Valverde, G. A. Houang, R. I. \& Wiley, D. E. (1997). Many visions, many aims. Cross National Investigation of Curricular Intentions in School Mathematics. Londres: Kluwer

SCHOENFELD, A. H. (1991). On mathematics as sense-making: An informal attack on the unfortunate divorce of formal and informal mathematics. En J. F. Voss, D. N. Perkins \& J. W. Segal (Eds.), Informal reasoning and education (pp. 311-343). Hillsdale, NJ: Lawrence Erlbaum Associates.

Stigler, J. W., FusOn, K., HAM, M. \& KIM, M, (1986). An analysis of addition and subtraction word problems in U.S. and Soviet elementary mathematics textbooks. Cognition and instruction, 3, 153-171

STRAY, C. (1994). Paradigms regained: towards a historical sociology of the textbook. Journal of Curriculum Studies, 26, 1-26.

VAN LIESHOUT, E. C., VERDWAALD, A. \& VAN HERK, J. (1997). Suppression of real-world knowledge and demand characteristics in word problem solving. Comunicación presentada en la Seventh European Conference on Learning and Instruction, Atenas, Grecia, Agosto.

VerschafFel, L. \& De Corte, E. (1997a). Word problems: A vehicle for authentic mathematical understanding and problem solving in the primary school? En T. Nunes \& P. Bryant (Eds.), Learning and teaching mathematics: An international perspective (pp. 69-98). Hove, UK: Psychology Press.

VersCHAFFel, L. \& DE CORTE, E. (1997b). Teaching realistic mathematical modeling and problem solving in the elementary school: A teaching experiment with fifth graders. Journal for Research in Mathematics Education, 28, 577-601. 
Verschaffel, L., De Corte, E. \& Borghart, L. (1997). Pre-service teachers' conceptions and beliefs about the role of realworld knowledge in mathematical modelling of school word problems. Learning and Instruction, 4, 339-359.

VerschafFel, L., De Corte, E. \& LASURE, S. (1994). Realistic considerations in mathematical modelling of school arithmetic word problems. Learning and Instruction, 4, 273-294.

Verschaffel, L., De CORTE, E. \& LASURe, S. (1999). Children's conceptions about the role of real-world knowledge in mathematical modeling of school word problems. En W. Schnotz, S. Vosniadou \& M. Carretero (Eds.), New perspectives on conceptual change (pp 175-189). Oxford: Elsevier.

Verschaffel, L., De Corte, E., Lasure, S. , van Vaerenbergh, G., Bogaerts, H. \& Ratinckx, E. (1999). Design and evaluation of a learning environment for mathematical modeling and problem solving in upper elementary school children. Mathematical Thinking and Learning, 1, 195-229.

VerschafFel, L., GreER, B. \& DE CORTE, E. (2000). Making sense of word problems. Lisse: Swets \& Zeitlinger.

WYNDHAMN, J. \& SÄLJÖ, R. (1997). Word problems and mathematical reasoning: A study of children's mastery of reference and meaning in textual realities. Learning and Instruction, 7, 361-382.

Yoshida, H., Verschaffel, L. \& De CorTe, E. (1997). Realistic considerations in solving problematic word problems: Do Japanese and Belgian children have the same difficulties? Learning and Instruction, 7, 329-338. 